\title{
Effect of treatment time on characterization and properties of nanocrystalline surface layer in copper induced by surface mechanical attrition treatment
}

\author{
FARZAD KARGAR*, M LALEH, T SHAHRABI and A SABOUR ROUHAGHDAM \\ Department of Materials Engineering, Faculty of Engineering, Tarbiat Modares University, \\ P.O. Box 14115-143, Tehran, Iran
}

MS received 23 June 2013; revised 28 October 2013

\begin{abstract}
Nanocrystalline surface layers were synthesized on pure copper by means of surface mechanical attrition treatment (SMAT) at various treatment times. The microstructural features of the surface layers produced by SMAT were systematically characterized by optical microscopy (OM), transmission electron microscopy (TEM) and X-ray diffraction (XRD) analysis. Hardness and surface roughness measurements were also carried out. It is found that the thickness of the deformed layer increased from 50 to $500 \mu \mathrm{m}$ with increasing treatment time from 10 to $300 \mathrm{~min}$, while the average grain size of the top surface layer decreased from 20 to $7 \mathrm{~nm}$. Hardness of the all SMATed samples decreased with depth. Furthermore, the hardness of the top surface layer of the SMATed samples was at least two times higher than that of the un-treated counterpart. Surface roughness results showed different trend with treatment time. Amounts of PV and $R_{a}$ values first sharply increased and then decreased.
\end{abstract}

Keywords. Nanocrystalline; copper; surface mechanical attrition treatment; hardness; surface roughness.

\section{Introduction}

It is well known that the majority of failure modes of engineering materials such as wear and corrosion are highly sensitive to the microstructure and properties of the materials surface. Accordingly, in most cases, material failures initiate at the surface. So, surface modification is expected to be a good and effective way to optimize the surface characteristics of the materials. Nanostructured materials (with grain size $<100 \mathrm{~nm}$ ) have been the subject of the widespread investigations over the past two decades because of their superior properties such as high strength, hardness and diffusivity in comparison with those coarse grain types (Lu et al 1991; Wei et al 2008). Nanocrystalline materials can be synthesized by various techniques such as inert gas condensation (IGC) (Gleiter 1989), mechanical alloying (MA) (Suryanarayana 2001), electrodeposition (Lu et al 2005; Shen et al 2005), crystallization from amorphous materials (Sun and Lu 1997) and severe plastic deformation. Among these techniques, severe plastic deformation has been widely used in order to form nanocrystalline materials (Wu et al 2002; Meyers et al 2006). Although any means of introducing large plastic strains in metals may lead to grain refinement, two principal methods for subjecting a material to severe plas-

*Author for correspondence (farzad.kargar@gmail.com) tic deformation have gained more acceptance, i.e. equalchannel angular pressing (ECAP) (Iwahashi et al 1996) and surface mechanical attrition treatment (SMAT). Experimental results indicate that the more common grain size of materials after ECAP process is about $200 \mathrm{~nm}$, but grains as small as $50 \mathrm{~nm}$ can be reached in Al alloys. An alternative procedure to introduce high plastic strains is SMAT. This is a recently developed method that can induce grain refinement into the nanometer regime on the surface of the bulk materials. The basic principle of the treatment is generation of high plastic deformation on the top surface layer of the specimen by means of repeated multidirectional and random collision of flying stainless steel balls ( $\mathrm{Lu}$ and Lu 1999; Wang et al 2003; Lu and Lu 2004; Revesz and Takacs 2007; Blonde et al 2010). SMAT has some benefits relative to other surface nanocrystallization methods. For example, it does not result in a difference in chemical composition between the nanocrystalline surface layer and matrix. Moreover, it is a simple, flexible and low-cost method. Indeed, SMAT manufactures contamination-free and porous-free samples (Zhang et al 2003; Lu et al 2006; Wen et al 2008). In recent years, the mechanism of surface nanocrystallization by SMAT process has been systematically investigated in pure metals, i.e. iron (Tao et al 2002; Wang et al 2003), cobalt (Wu et al 2005, 2007), titanium (Guo et al 2004; Wen et al 2007, 2008), copper (Wang et al 2003, 2010; Li et al 2008), zirconium (Zhang et al 2008; Zhang 
and Han 2009), Ni (Wei et al 2009) and alloys, i.e. Al alloys (Wu et al 2002; Dai et al 2004, 2005), AISI 316 stainless steel (Lu et al 2006; Roland et al 2006), AISI 304 stainless steel (Zhang et al 2003), AZ91D magnesium alloy (Sun et al 2008a, b; Wei et al 2008), NiTi alloy (Hu et al 2009), and $\mathrm{Ni}_{3} \mathrm{Al}$ alloy (Ren et al 2006). These investigations have demonstrated that the grain refinement mechanism depends strongly on the lattice structure and stacking fault energy (SFE) of the treated material, and on the other hand, processing parameters, including the treatment time, diameter of the balls and vibration frequency used in the treatment. We emphasize that there is a lack of literature about the effect of the treatment time on the properties of the refined layer, particularly in the long term. In the present study, pure copper was used as the initial material. In order to investigate the effect of the treatment time on the properties of the surface layer, SMAT was performed at various periods of time. Nanocrystalline structures were characterized by different techniques, and the coarse grain copper was used for comparison.

\section{Experimental}

A commercial purity copper (99.9 wt\%) disc (30 mm in diameter and $5 \mathrm{~mm}$ in thickness) was used as the initial sample in this experiment. Prior to SMAT, the copper samples were annealed at $923 \mathrm{~K}$ for $2 \mathrm{~h}$ to obtain homogeneous coarse-grained structure. Nanocrystallization of the samples was carried out by using SMAT treatment. Schematic of the SMAT set-up used in this study is shown in figure 1 . The surface of the bulk samples was subjected to periodic bombardments of stainless steel balls ( $8 \mathrm{~mm}$ in diameter) in a vacuum chamber at various times (10, 30, 60, 120, 180 and $300 \mathrm{~min})$ at a vibrating frequency of $50 \mathrm{~Hz}$. An optical microscopy (Olympus BX51M) was used to observe the microstructural development along a section perpendicular to the treated surface of the samples. Before microstructural observation, the samples were electrolytically etched in $10 \mathrm{wt} \%$ phosphoric acid. Microstructural feature in the surface layer of the SMATed sample (treated for $300 \mathrm{~min}$ ) was characterized by using a Philips EM-420 transmission electron microscope (TEM) (Philips Electronics, Amsterdam, The Netherlands), operating at a voltage of $120 \mathrm{kV}$. Thin foil samples for TEM observations were prepared first by mechanically polishing the specimen on the untreated side until it was about $30 \mu \mathrm{m}$ thick followed by Ar ion milling at low temperatures. Average grain size of the top surface layer of the SMATed samples was obtained from X-ray diffraction (XRD) line broadening. XRD experiments were performed on a Siemens D-500 diffractometer with $\mathrm{CuK} \alpha$ radiation in the range of $30-80^{\circ}$ with a step width of $0.02^{\circ}$. Vickers microhardness (HV) tests on the cross sections of the samples were carried out on a Buhler microtest device with an applied load of $0 \cdot 1 \mathrm{~N}$ for $10 \mathrm{~s}$. The microhardness variation was measured along the depth (up to $600 \mu \mathrm{m}$ from the top surface), and the distance between any two neighbouring indentations was at least $10 \mu \mathrm{m}$. The average surface roughness $\left(R_{\mathrm{a}}\right)$ and peakto-valley (PV) of the copper samples before and after SMAT was measured using a stylus type surface profilometer (Taylor Hobson Surtronic 25) with an accuracy of $0 \cdot 01 \mu \mathrm{m}$.

\section{Results and discussion}

Figure 2 shows the cross-sectional optical microscopic microstructures of the as-received and SMATed copper samples. As can be seen, the as-received sample has coarse grains with grain size from 50 to $100 \mu \mathrm{m}$. After SMAT, the coarse grains disappeared and severely deformed surface layers were produced. Obviously, the plastic deformation along the depth for all SMATed samples is inhomogeneous; i.e. irregular, multiple slips near the top surface were gradually replaced by single slips with increasing depth. During SMAT, the balls provided repeated multidirectional impacts on a surface at a high speed and generated high-density dislocations and deformation twins. The resultant dislocations could be re-arranged under stress to the dislocation networks which led to the formation of nanocrystallites separated by grain boundaries. Furthermore, deformation twins subdivided the original grains efficiently by introducing different boundaries and leading to formation of refined microstructures. The formation of nano-sized grains is mainly due to three factors: a very large strain, an extremely large strain rate and repetitive loading by balls impacts. Mechanism of grain refinement in pure $\mathrm{Cu}$ was systematically studied by Wang et al (2006). They found

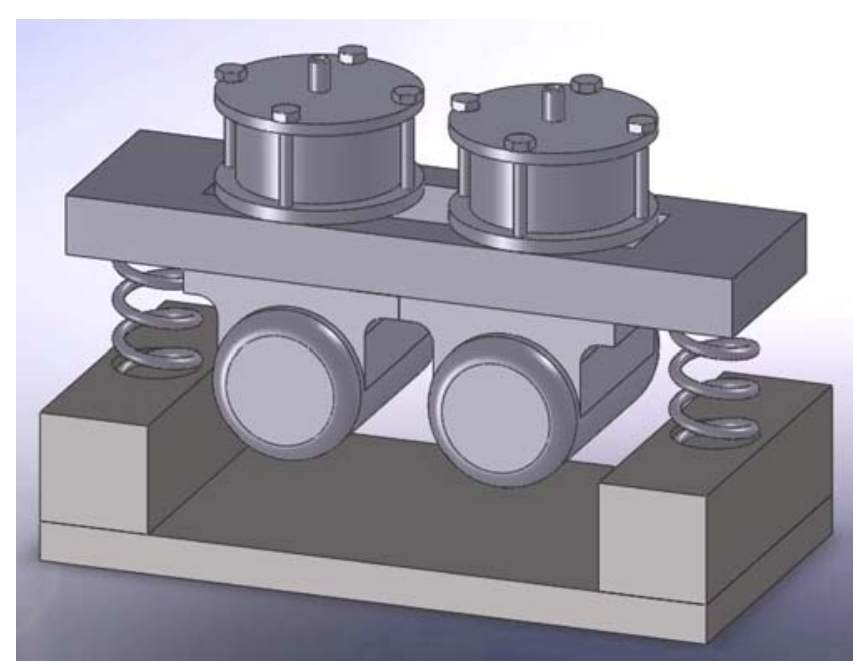

Figure 1. Schematic of the surface mechanical attrition treatment set-up used in this study. 

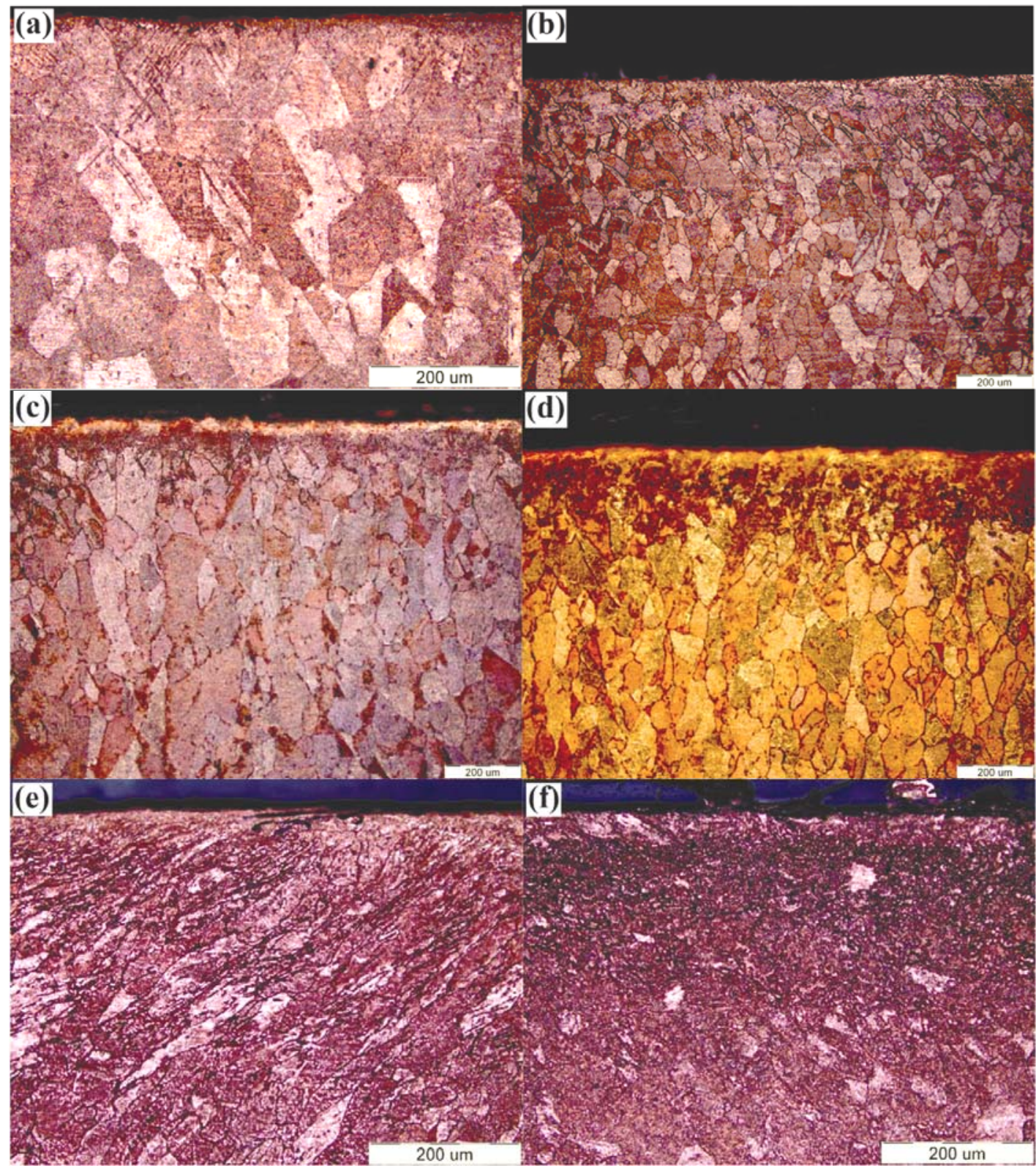

Figure 2. Cross-sectional microstructure of the SMATed samples after different treating times: (a) 10, (b) 30, (c) 60, (d) 120, (e) 180 and (f) $300 \mathrm{~min}$.

that the plastic strain-induced grain refinement in copper closely relates to its deformation modes, and identified two different mechanisms corresponding to different levels of strain rate. In the subsurface layer (thickness $>25 \mu \mathrm{m}$ ) of the SMATed $\mathrm{Cu}$, where strain rate is low and deformation is mainly dominated by dislocation activities, grains in the submicron scales are obtained via formation and development of dislocation cells. The grain refinement process includes: (i) formation of dislocation cells in original grains; (ii) transformation of dislocation walls into sub-boundaries with small misorientations separating the original grains into individual subgrains; (iii) evolution of sub-boundaries into highly misoriented grain boundaries. On the top surface layer (few microns beneath the uppermost surface) of the SMATed $\mathrm{Cu}$, strain rates are much increased so that deformation twining plays a significant role in plastic deformation. The grain refinement process involves formation of high-density nanometer-thick twins dividing the original coarse grains to twin-matrix lamellae, development of dislocation walls that leads to further division of the twin-matrix lamellae into equiaxed nano-sized blocks, and evolution of these preferentially oriented blocks into randomly oriented grains (Wang et al 2006). Grains cannot be observed in the regions near the treated surface (beneath the top surface) with optical microscopy. Figure 3(a) shows a 

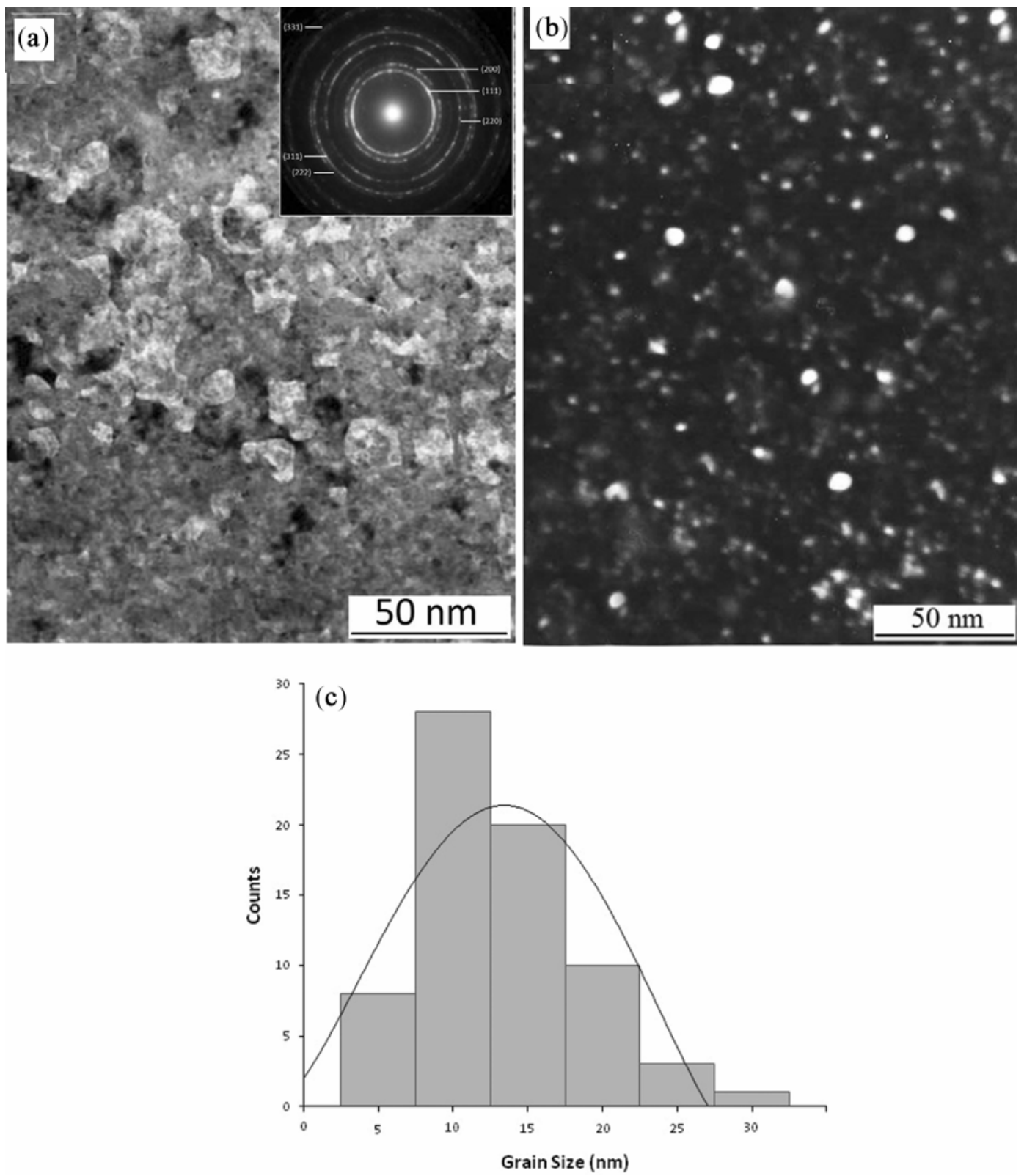

Figure 3. (a) Bright field TEM image and corresponding selected area diffraction (SAD) pattern (the inset), (b) dark field TEM image and (c) grain size distribution of the top-treated surface layer of the SMATed copper sample (treated for $300 \mathrm{~min}$ ).

typical TEM plane view observation and selected area diffraction (SAD) pattern (inset) of the top surface layer in the SMATed copper sample (treated for $300 \mathrm{~min}$ ). It is clear that the microstructure of the top-treated surface layer is characterized by ultrafine equiaxed grains with random crystallographic orientations. As can be seen, the mean grain size in the surface layer is $\sim 10 \mathrm{~nm}$. Quantitative analysis of dark-field TEM image shows that the plane-view grain size of the sample ranges from 3 to $30 \mathrm{~nm}$ with an average value of $12 \pm 3 \mathrm{~nm}$ (as shown in figure 3c). XRD patterns of the $\mathrm{Cu}$ samples before and after SMAT are shown in figure 4(a). It is observed that after SMAT, there is an evident broadening of the Bragg reflections relative to the coarse-grained sample. This can be mainly attributed to grain refinement, increase in the atomic-level strain and micro-distortions of the crystalline lattice. Only a slight change can be observed in the XRD patterns with increasing SMAT duration. The average grain size of the surface layer after SMAT was calculated using Scherrer-Wilson equation. Figure 4(b) shows the variation of grain size and thickness of the deformed layer as a function of treating time, which were obtained from XRD and optical microscopy analyses, respectively. Results show that the average grain size of the top 

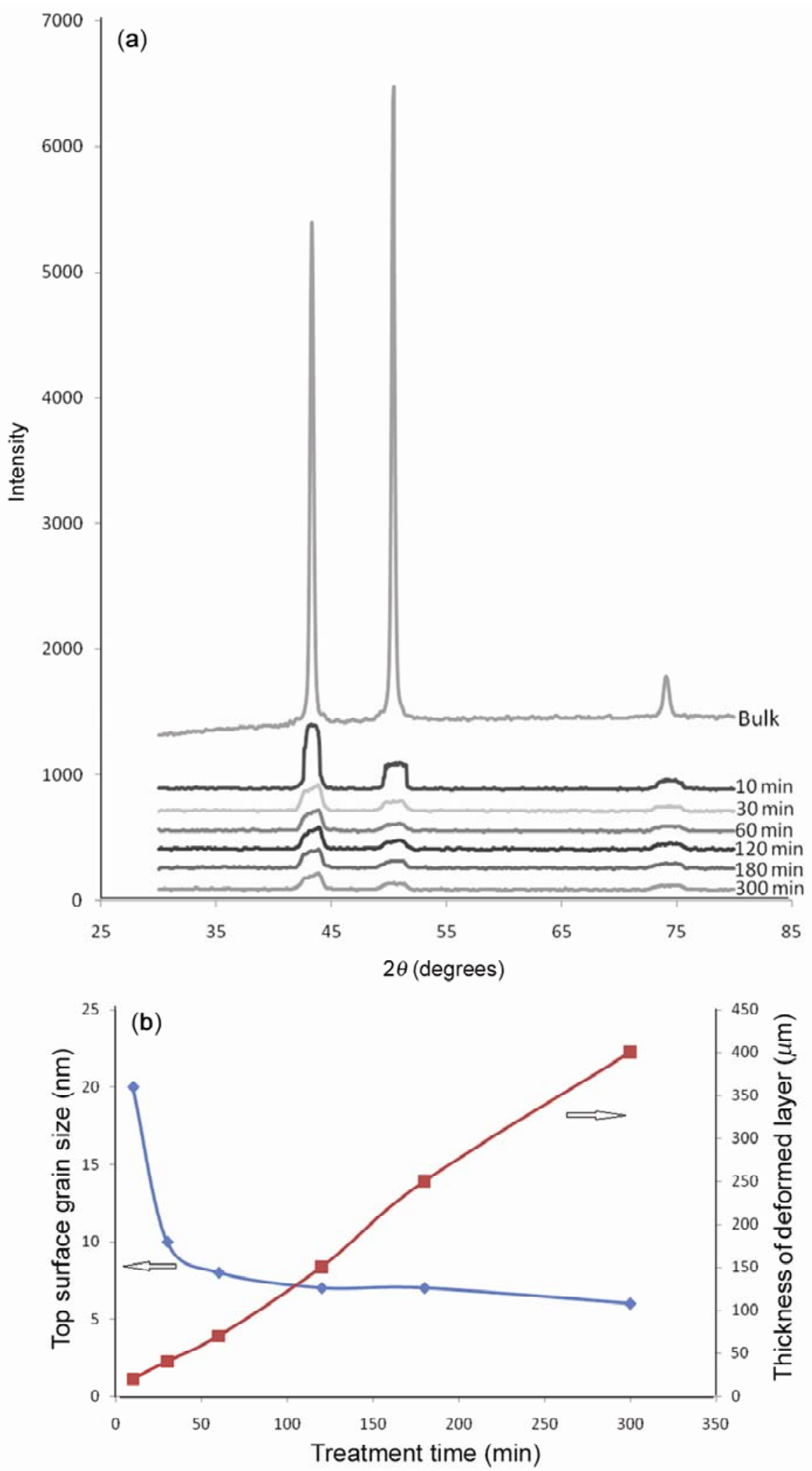

Figure 4. (a) XRD patterns of the copper samples before and after SMAT at different times. (b) Variation of the grain size and thickness of the deformed layer of the SMATed samples as a function of time duration measured from XRD patterns and microscopic observations, respectively. 

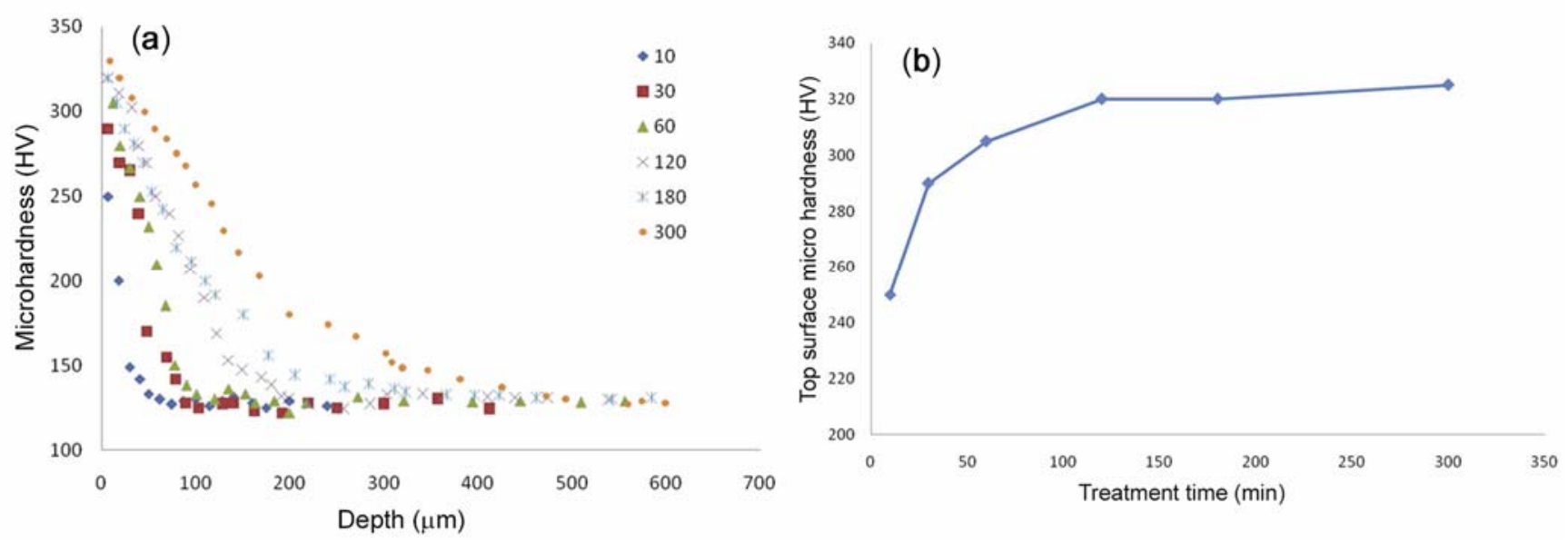

Figure 5. (a) Variation of hardness with depth from the top surface on a cross section of the SMATed samples. (b) Variation of hardness of the top surface layer of the SMATed samples with treating time.

surface layer of the SMATed samples reduced gradually with treating time. However, the thickness of the severely deformed layer increased obviously with treating time. These realities suggest that at short treating times (up to $120 \mathrm{~min})$, increase in the treatment time leads to more reduction in grain size (from 20 to $8 \mathrm{~nm}$ ), but further increase in the treating time (from 120 to $300 \mathrm{~min}$ ) has not shown any significant change in the grain size. Figure 5(a) shows the microhardness variation with the distance from the SMATed top surface to the matrix for samples treated at different times. The hardness value decreases gradually with farther distance from the surface. The diffusion zone depths (defined by the distance from the top surface to the point where the microhardness is same with that of the matrix) are different for SMATed samples, and increase with treatment time, which are also in good agreement with the microscopic observations shown in figure 2. The microhardness values of the top surface of the SMATed sample as a function of grain size is shown in figure 5(b). A sharp increase in the hardness is obtained after treatment for $10 \mathrm{~min}$. However, with further increase in the treatment time, it is found that the hardness values of the top surface of the SMATed samples increase slightly and these values are at least two times higher than that of un-treated copper. According to the well-known Hall-Petch relationship, large grain refinement into nanometer scale that is induced by SMAT can lead to strengthening and hardening of the copper samples. Based on the microhardness results, it can be concluded that the increment in the treatment time did not show significant effect on the top surface hardness. Because the grain size of the top layer of the SMATed samples is more similar in the long period of treatment times (i.e. 120, 180 and $300 \mathrm{~min}$ ), the hardness values do not show any obvious change. It becomes interesting that nanocrystalline can also be found even for very short duration (i.e. $10 \mathrm{~min}$ ). This short-treatment duration has important meaning in engineering applications because it can save time and energy. There is a wide range of numerical parameters and measurement methods available for characterization of surface roughness. However, heightbased parameters have been commonly used in basic roughness characterizations because they can easily be determined from stylus-type profilometer. The arithmetic mean roughness ( $R_{\mathrm{a}}$ value) is defined as the average of the absolute value of all area values, $y(x)$, contained within a profile length, as shown in (1)

$$
R_{\mathrm{a}}=\frac{1}{L} \int_{0}^{L}|Z(x)| \mathrm{d} x
$$

another parameter used for roughness measurements is the vertical distance between the highest peak and the lowest valley (PV) of the profile. The $R_{\mathrm{a}}$ and PV values were used in this study because they have been used routinely in industrial applications to quantify the changes in the surface morphology and they are reasonable representations of the roughening behaviour of the materials. The relationship between grain size and roughness is often found to be linear (Mahmudi and Mehdizadeh 1998), but some investigations show deviations from the linear behaviour, especially at higher strains (Dai et al 2004, 2005). The changes of $R_{\mathrm{a}}$ and PV of the SMATed samples as a function of treating time are shown in figure 6 . It should be noted that the data points shown in these curves are the average values of the three traces. Both curves indicate that the grain size has an appreciable effect on the roughening behaviour of the SMATed samples. As can be clearly seen from both the curves, with increase in the treatment duration, the amount of PV and $R_{\mathrm{a}}$ values first sharply increase and then decrease. At first, when the balls are impacted to the smooth surface, the indents created by the impacting balls are separated. Each of these indents generates a pair of the peak and valley, 


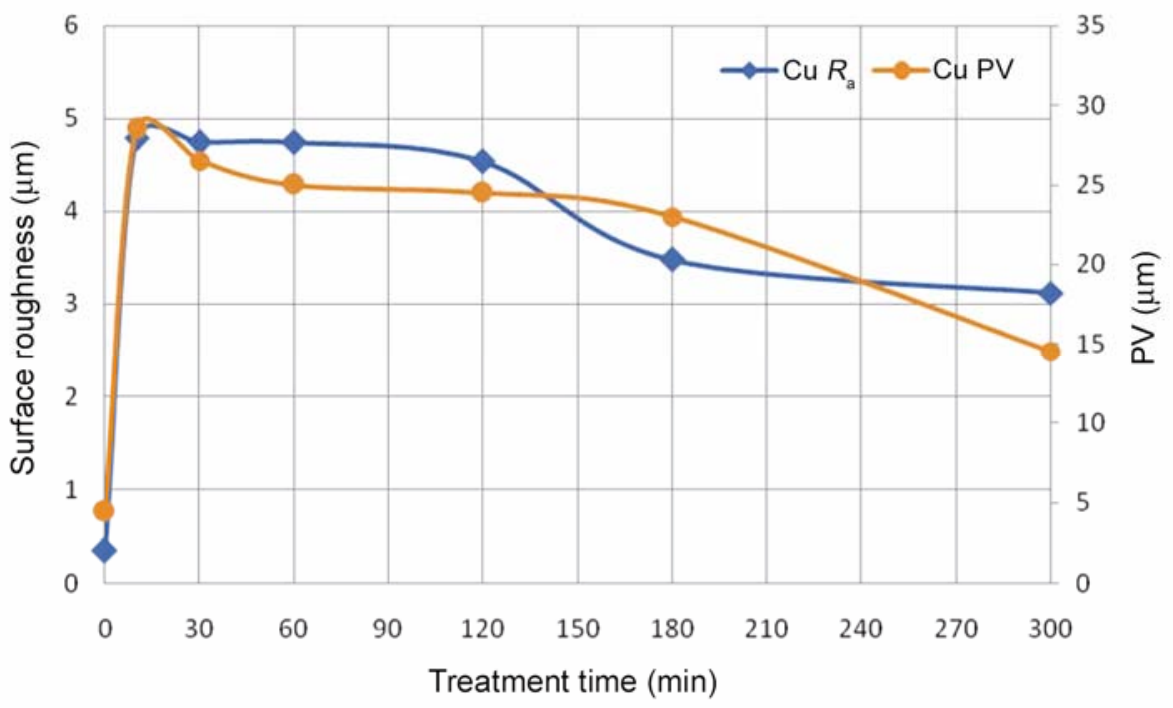

Figure 6. Variation of $R_{\mathrm{a}}$ and $\mathrm{PV}$ as a function of SMAT time.

so the PV value rises as a result of the formation of new indents. But, by increasing the treatment time from 10 to $30 \mathrm{~min}$, the amount of the indents increases and, as a consequence, the PV value decreases because of the reduction of the smooth contact area and the increment of the surface hardness (increased resistance to plastic deformation). By further increasing the treatment duration, the PV value change is small while it keeps its reducing trend. The trend and evolution sequence of the $R_{\mathrm{a}}$ value can be deduced from the investigation of the PV value.

\section{Conclusions}

Nanocrystalline surface layers were successfully synthesized on pure copper by means of SMAT at different treatment times. Results showed that the treatment time has a significant effect on characterization and properties of surface layers. Thickness of the deformed layer increased from 50 to $500 \mu \mathrm{m}$ with increasing duration from 10 to $300 \mathrm{~min}$. Average grain size of the top surface layer of the SMATed samples decreased from 20 to $7 \mathrm{~nm}$ with time. As a result of grain refinement, the hardness of the SMATed samples increased by a factor of two or so, and the hardness value decreased with increasing depth from the top surface. Surface roughness results showed in a different manner, i.e. the $R_{\mathrm{a}}$ and $\mathrm{PV}$ values, first increased sharply and then decreased.

\section{References}

Blonde R, Chan H, Allian-Bonasso N, Bolle B, Grosdidier T and Lu J 2010 J. Alloys. Compd. 504S S410

Dai K, Villegas J and Shaw L 2005 Scr. Mater. 52259
Dai K, Villegas J, Stone Z and Shaw L 2004 Acta Mater. 52 5771

Gleiter H 1989 Prog. Mater. Sci. 33223

Guo F A, Zhu Y K, Trannoy N and Lu J 2004 Thermochim. Acta 419239

Hu T, Wen C S, Lu J, Wu S L, Xin Y C, Zhang W J, Chu C L, Chung J C Y, Yeung K W K, Kowk D T K and Chu P K 2009 J. Alloys Compd. 482298

Iwahashi Y, Wang J, Horita Z, Nemoto M and Langdon T G 1996 Scr. Mater. 35143

Li W L, Tao N R and Lu K 2008 Scr. Mater. 59546

Lu A Q, Zhang Y, Li Y, Liu G, Zang Q H and Liu C M 2006 Acta Metal. Sin. 19183

Lu K and Lu J 2004 Mater. Sci. Eng. A375 38

Lu K and Lu J 1999 J. Mater. Sci. Technol. 15193

Lu K, Wang J T and Wei W D 1991 J. Appl. Phys. 69 522

Lu L, Schwaiger R, Shan Z W, Dao M, Lu K and Suresh S 2005 Acta Mater. 532169

Mahmudi R and Mehdizadeh M 1998 J. Mater. Process. Technol. 80-81 707

Meyers M A, Mishra A and Benson D J 2006 Prog. Mater. Sci. 51427

Ren J, Shan A, Zhang J, Song H and Liu J 2006 Mater. Lett. 60 2076

Revesz A and Takacs L 2007 J. Alloys Compd. 441111

Roland T, Retraint D, Lu K and Lu J 2006 Scr. Mater. 54 1949

Shen Y F, Lu L, Lu Q H, Jin Z H and Lu K 2005 Scr. Mater. 52 989

Sun H Q, Shi Y N and Zhang M X 2008a Surf. Coat. Technol. 2022859

Sun H Q, Shi Y N, Zhang M X and Lu K 2008b Surf. Coat. Technol. 2023947

Sun N X and Lu K 1997 Phys. Rev. B56 5885

Suryanarayana C 2001 Prog. Mater. Sci. 461

Tao N R, Wang Z B, Tong W P, Sui M L, Lu J and Lu K 2002 Acta Mater. 504603 
Wang K, Tao N R, Liu G, Lu J and Lu K 2006 Acta Mater. 54 5281

Wang Y M, Wang K, Pan D, Lu K, Hemker K J and Ma E 2003 Scr. Mater. 481581

Wang Z B, Lu K, Wilde G and Divinski S V 2010 Acta Mater. 582376

Wang Z B, Tao N R, Tong W P, Lu J and Lu K 2003 Acta Mater. $\mathbf{5 1} 4319$

Wei L, Ping L, Fengcang M and Yonghua R 2009 J. Mater. Sci. 442925

Wei Y, Liu B, Hou Li, Xu B and Liu G 2008 J. Alloys Compd. 452336

Wen C, Li W and Rong Y 2008 Mater. Sci. Eng. A481 484
Wen M, Gu J, Liu G, Wang Z and Lu J 2007 Surf. Coat. Technol. 2016285

Wen M, Liu G, Gu J F, Guan W M and Lu J 2008 Surf. Coat. Technol. 2024728

Wu X, Tao N, Hong Y, Liu G, Xu B, Lu J and Lu K 2005 Acta Mater. 53681

Wu X, Tao N, Hong Y, Xu B, Lu J and Lu K 2002 Acta Mater. 502075

Wu X L, Tao N R, Wei Q M, Jiang P, Lu J and Lu K 2007 Acta Mater. 555768

Zhang H W, Hei Z K, Liu G, Lu J and Lu K 2003 Acta Mater. 511871

Zhang L and Han Y 2009 Mater. Sci. Eng. A523 130

Zhang L, Han Y and Lu J 2008 Nanotechnology 19165706 